EUROPEAN LABORATORY FOR PARTICLE PHYSICS

CERN-EP/98-090

4th June 1998

\title{
Measurement of Tau Branching Ratios to Five Charged Hadrons
}

\author{
The OPAL Collaboration
}

\begin{abstract}
The branching ratios of the decay of the $\tau$ lepton to five charged hadrons have been measured with the OPAL detector at LEP using data collected between 1991 and 1995 at $\mathrm{e}^{+} \mathrm{e}^{-}$centre-of-mass energies close to the $\mathrm{Z}^{0}$ resonance. The branching ratios are measured to be

$$
\begin{gathered}
B\left(\tau^{-} \rightarrow 3 h^{-} 2 h^{+} \nu_{\tau}\right)=(0.091 \pm 0.014 \pm 0.005) \% \\
B\left(\tau^{-} \rightarrow 3 h^{-} 2 h^{+} \pi^{0} \nu_{\tau}\right)=(0.027 \pm 0.018 \pm 0.007) \%
\end{gathered}
$$

where the first error is statistical and the second systematic.
\end{abstract}

(Submitted to the European Physical Journal) 


\section{The OPAL Collaboration}

K. Ackerstaff ${ }^{8}$, G. Alexander ${ }^{23}$, J. Allison ${ }^{16}$, N. Altekamp ${ }^{5}$, K.J. Anderson ${ }^{9}$, S. Anderson ${ }^{12}$, S. Arcelli ${ }^{2}$, S. Asai ${ }^{24}$, S.F. Ashby ${ }^{1}$, D. Axen ${ }^{29}$, G. Azuelos ${ }^{18, a}$, A.H. Ball ${ }^{17}$, E. Barberio ${ }^{8}$, R.J. Barlow ${ }^{16}$, R. Bartoldus ${ }^{3}$, J.R. Batley ${ }^{5}$, S. Baumann ${ }^{3}$, J. Bechtluft ${ }^{14}$, T. Behnke ${ }^{8}$, K.W. Bell ${ }^{20}$, G. Bella ${ }^{23}$, S. Bentvelsen ${ }^{8}$,

S. Bethke ${ }^{14}$, S. Betts ${ }^{15}$, O. Biebel ${ }^{14}$, A. Biguzzi ${ }^{5}$, S.D. Bird ${ }^{16}$, V. Blobel ${ }^{27}$, I.J. Bloodworth ${ }^{1}$, M. Bobinski ${ }^{10}$, P. Bock ${ }^{11}$, J. Böhme ${ }^{14}$, M. Boutemeur ${ }^{34}$, S. Braibant ${ }^{8}$, P. Bright-Thomas ${ }^{1}$, R.M. Brown ${ }^{20}$, H.J. Burckhart ${ }^{8}$, C. Burgard ${ }^{8}$, R. Bürgin ${ }^{10}$, P. Capiluppi ${ }^{2}$, R.K. Carnegie ${ }^{6}$, A.A. Carter $^{13}$, J.R. Carter ${ }^{5}$, C.Y. Chang ${ }^{17}$,

D.G. Charlton ${ }^{1, b}$, D. Chrisman ${ }^{4}$, C. Ciocca ${ }^{2}$, P.E.L. Clarke ${ }^{15}$, E. Clay ${ }^{15}$, I. Cohen ${ }^{23}$, J.E. Conboy ${ }^{15}$, O.C. Cooke ${ }^{8}$, C. Couyoumtzelis ${ }^{13}$, R.L.Coxe ${ }^{9}$, M. Cuffiani ${ }^{2}$, S. Dado ${ }^{22}$, G.M. Dallavalle ${ }^{2}$, R. Davis ${ }^{30}$, S. De Jong ${ }^{12}$, L.A. del Pozo ${ }^{4}$, A. de Roeck ${ }^{8}$, K. Desch ${ }^{8}$, B. Dienes ${ }^{33, d}$, M.S. Dixit ${ }^{7}$, M. Doucet ${ }^{18}$, J. Dubbert ${ }^{34}$, E. Duchovni ${ }^{26}$, G. Duckeck ${ }^{34}$, I.P. Duerdoth ${ }^{16}$, D. Eatough $^{16}$, P.G. Estabrooks ${ }^{6}$, E. Etzion ${ }^{23}$, H.G. Evans ${ }^{9}$,

F. Fabbri ${ }^{2}$, A. Fanfani ${ }^{2}$, M.Fanti ${ }^{2}$, A.A.Faust ${ }^{30}$, F. Fiedler ${ }^{27}$, M. Fierro ${ }^{2}$, H.M. Fischer ${ }^{3}$, I. Fleck ${ }^{8}$, R. Folman ${ }^{26}$, A. Fürtjes ${ }^{8}$, D.I. Futyan ${ }^{16}$, P. Gagnon ${ }^{7}$, J.W. Gary ${ }^{4}$, J. Gascon ${ }^{18}$, S.M. Gascon-Shotkin ${ }^{17}$, C. Geich-Gimbel ${ }^{3}$, T. Geralis ${ }^{20}$, G. Giacomelli ${ }^{2}$, P. Giacomelli ${ }^{2}$, V. Gibson ${ }^{5}$, W.R. Gibson ${ }^{13}$,

D.M. Gingrich ${ }^{30, a}$, D. Glenzinski ${ }^{9}$, J. Goldberg ${ }^{22}$, W. Gorn ${ }^{4}$, C. Grandi ${ }^{2}$, E. Gross ${ }^{26}$, J. Grunhaus ${ }^{23}$,

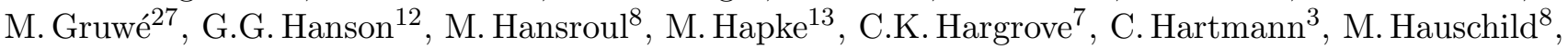

C.M. Hawkes ${ }^{5}$, R. Hawkings ${ }^{27}$, R.J.Hemingway ${ }^{6}$, M. Herndon ${ }^{17}$, G. Herten ${ }^{10}$, R.D. Heuer ${ }^{8}$,

M.D. Hildreth ${ }^{8}$, J.C.Hill ${ }^{5}$, S.J.Hillier ${ }^{1}$, P.R. Hobson ${ }^{25}$, A. Hocker ${ }^{9}$, R.J. Homer ${ }^{1}$, A.K. Honma ${ }^{28, a}$, D. Horváth ${ }^{32, c}$, K.R. Hossain ${ }^{30}$, R. Howard ${ }^{29}$, P. Hüntemeyer ${ }^{27}$, P. Igo-Kemenes ${ }^{11}$, D.C. Imrie ${ }^{25}$, K. Ishii ${ }^{24}$, F.R. Jacob ${ }^{20}$, A. Jawahery ${ }^{17}$, H. Jeremie ${ }^{18}$, M. Jimack ${ }^{1}$, A. Joly ${ }^{18}$, C.R. Jones ${ }^{5}$, P. Jovanovic ${ }^{1}$, T.R. Junk ${ }^{8}$, D. Karlen ${ }^{6}$, V. Kartvelishvili ${ }^{16}$, K. Kawagoe ${ }^{24}$, T. Kawamoto ${ }^{24}$, P.I. Kayal ${ }^{30}$, R.K. Keeler ${ }^{28}$, R.G. Kellogg ${ }^{17}$,

B.W.Kennedy ${ }^{20}$, A. Klier ${ }^{26}$, S.Kluth ${ }^{8}$, T. Kobayashi' ${ }^{24}$, M.Kobel ${ }^{3, e}$, D.S. Koetke ${ }^{6}$, T.P. Kokott ${ }^{3}$,

M. Kolrep ${ }^{10}$, S. Komamiya ${ }^{24}$, R.V.Kowalewski ${ }^{28}$, T. Kress ${ }^{11}$, P. Krieger ${ }^{6}$, J. von Krogh ${ }^{11}$, P. Kyberd ${ }^{13}$, G.D. Lafferty ${ }^{16}$, D. Lanske ${ }^{14}$, J. Lauber ${ }^{15}$, S.R. Lautenschlager ${ }^{31}$, I. Lawson ${ }^{28}$, J.G. Layter ${ }^{4}$, D. Lazic ${ }^{22}$,

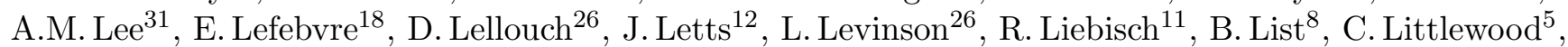

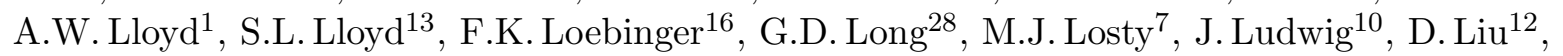

A. Macchiolo ${ }^{2}$, A. Macpherson ${ }^{30}$, M. Mannelli ${ }^{8}$, S. Marcellini $^{2}$, C. Markopoulos ${ }^{13}$, A.J. Martin ${ }^{13}$, J.P. Martin ${ }^{18}$, G. Martinez ${ }^{17}$, T. Mashimo ${ }^{24}$, P. Mättig ${ }^{26}$, W.J. McDonald ${ }^{30}$, J. McKenna ${ }^{29}$,

E.A. Mckigney ${ }^{15}$, T.J. McMahon ${ }^{1}$, R.A. McPherson ${ }^{28}$, F. Meijers ${ }^{8}$, S. Menke ${ }^{3}$, F.S. Merritt ${ }^{9}$, H. Mes ${ }^{7}$, J. Meyer $^{27}$, A. Michelini ${ }^{2}$, S. Mihara ${ }^{24}$, G. Mikenberg ${ }^{26}$, D.J. Miller ${ }^{15}$, R. Mir ${ }^{26}$, W. Mohr ${ }^{10}$, A. Montanari ${ }^{2}$, T. Mori ${ }^{24}$, K. Nagai ${ }^{26}$, I. Nakamura ${ }^{24}$, H.A. Neal ${ }^{12}$, B. Nellen ${ }^{3}$, R. Nisius ${ }^{8}$, S.W. O'Neale ${ }^{1}$, F.G. Oakham ${ }^{7}$,

F. Odorici ${ }^{2}$, H.O. Ogren ${ }^{12}$, M.J. Oreglia ${ }^{9}$, S. Orito ${ }^{24}$, J. Pálinkás ${ }^{33, d}$, G. Pásztor ${ }^{32}$, J.R. Pater ${ }^{16}$, G.N. Patrick ${ }^{20}$, J.Patt ${ }^{10}$, R. Perez-Ochoa ${ }^{8}$, S.Petzold ${ }^{27}$, P. Pfeifenschneider ${ }^{14}$, J.E. Pilcher ${ }^{9}$, J. Pinfold ${ }^{30}$, D.E. Plane ${ }^{8}$, P. Poffenberger ${ }^{28}$, B. Poli ${ }^{2}$, J. Polok ${ }^{8}$, M. Przybycień ${ }^{8}$, C. Rembser ${ }^{8}$, H. Rick ${ }^{8}$, S. Robertson ${ }^{28}$, S.A. Robins ${ }^{22}$, N. Rodning ${ }^{30}$, J.M. Roney ${ }^{28}$, K. Roscoe ${ }^{16}$, A.M. Rossi ${ }^{2}$, Y. Rozen ${ }^{22}$, K. Runge ${ }^{10}$, O. Runolfsson ${ }^{8}$, D.R.Rust ${ }^{12}$, K. Sachs ${ }^{10}$, T.Saeki ${ }^{24}$, O. Sahr ${ }^{34}$, W.M. Sang ${ }^{25}$, E.K.G. Sarkisyan ${ }^{23}$, C. Sbarra ${ }^{29}$, A.D.Schaile ${ }^{34}$, O. Schaile ${ }^{34}$, F. Scharf ${ }^{3}$, P. Scharff-Hansen ${ }^{8}$, J. Schieck ${ }^{11}$, B. Schmitt ${ }^{8}$, S. Schmitt ${ }^{11}$, A.Schöning ${ }^{8}$, T. Schorner ${ }^{34}$, M.Schröder ${ }^{8}$, M. Schumacher ${ }^{3}$, C. Schwick ${ }^{8}$, W.G. Scott ${ }^{20}$, R.Seuster ${ }^{14}$, T.G. Shears ${ }^{8}$, B.C.Shen ${ }^{4}$, C.H.Shepherd-Themistocleous ${ }^{8}$, P. Sherwood ${ }^{15}$, G.P. Siroli ${ }^{2}$, A. Sittler ${ }^{27}$, A.Skuja ${ }^{17}$, A.M. Smith ${ }^{8}$, G.A. Snow ${ }^{17}$, R. Sobie $^{28}$, S. Söldner-Rembold ${ }^{10}$, M. Sproston ${ }^{20}$, A. Stahl ${ }^{3}$, K.Stephens ${ }^{16}$, J.Steuerer ${ }^{27}$, K. Stoll ${ }^{10}$, D. Strom ${ }^{19}$, R. Ströhmer ${ }^{34}$, L. Stumpf ${ }^{28}$, R. Tafirout ${ }^{18}$,

S.D. Talbot ${ }^{1}$, S. Tanaka ${ }^{24}$, P. Taras ${ }^{18}$, S. Tarem ${ }^{22}$, R. Teuscher ${ }^{8}$, M. Thiergen ${ }^{10}$, M.A. Thomson ${ }^{8}$, 
E. von Törne ${ }^{3}$, E. Torrence ${ }^{8}$, S. Towers $^{6}$, I. Trigger ${ }^{18}$, Z. Trócsányi ${ }^{33}$, E. Tsur $^{23}$, A.S. Turcot ${ }^{9}$, M.F. Turner-Watson ${ }^{8}$, R. Van Kooten ${ }^{12}$, P. Vannerem ${ }^{10}$, M. Verzocchi ${ }^{10}$, P. Vikas ${ }^{18}$, H. Voss ${ }^{3}$, F. Wäckerle ${ }^{10}$, A. Wagner ${ }^{27}$, C.P. Ward ${ }^{5}$, D.R. Ward $^{5}$, P.M. Watkins ${ }^{1}$, A.T. Watson ${ }^{1}$, N.K. Watson ${ }^{1}$, P.S. Wells ${ }^{8}$, N. Wermes ${ }^{3}$, J.S. White ${ }^{28}$, G.W.Wilson ${ }^{14}$, J.A. Wilson ${ }^{1}$, T.R. Wyatt $^{16}$, S. Yamashita ${ }^{24}$, G. Yekutieli ${ }^{26}$, V. Zacek ${ }^{18}$, D. Zer-Zion ${ }^{8}$

${ }^{1}$ School of Physics and Astronomy, University of Birmingham, Birmingham B15 2TT, UK

2Dipartimento di Fisica dell' Università di Bologna and INFN, I-40126 Bologna, Italy

${ }^{3}$ Physikalisches Institut, Universität Bonn, D-53115 Bonn, Germany

${ }^{4}$ Department of Physics, University of California, Riverside CA 92521, USA

${ }^{5}$ Cavendish Laboratory, Cambridge CB3 0HE, UK

${ }^{6}$ Ottawa-Carleton Institute for Physics, Department of Physics, Carleton University, Ottawa, Ontario K1S 5B6, Canada

${ }^{7}$ Centre for Research in Particle Physics, Carleton University, Ottawa, Ontario K1S 5B6, Canada

${ }^{8}$ CERN, European Organisation for Particle Physics, CH-1211 Geneva 23, Switzerland

${ }^{9}$ Enrico Fermi Institute and Department of Physics, University of Chicago, Chicago IL 60637, USA

${ }^{10}$ Fakultät für Physik, Albert Ludwigs Universität, D-79104 Freiburg, Germany

${ }^{11}$ Physikalisches Institut, Universität Heidelberg, D-69120 Heidelberg, Germany

${ }^{12}$ Indiana University, Department of Physics, Swain Hall West 117, Bloomington IN 47405, USA

${ }^{13}$ Queen Mary and Westfield College, University of London, London E1 4NS, UK

${ }^{14}$ Technische Hochschule Aachen, III Physikalisches Institut, Sommerfeldstrasse 26-28, D-52056 Aachen,

Germany

${ }^{15}$ University College London, London WC1E 6BT, UK

${ }^{16}$ Department of Physics, Schuster Laboratory, The University, Manchester M13 9PL, UK

${ }^{17}$ Department of Physics, University of Maryland, College Park, MD 20742, USA

${ }^{18}$ Laboratoire de Physique Nucléaire, Université de Montréal, Montréal, Quebec H3C 3J7, Canada

${ }^{19}$ University of Oregon, Department of Physics, Eugene OR 97403, USA

${ }^{20}$ Rutherford Appleton Laboratory, Chilton, Didcot, Oxfordshire OX11 0QX, UK

${ }^{22}$ Department of Physics, Technion-Israel Institute of Technology, Haifa 32000, Israel

${ }^{23}$ Department of Physics and Astronomy, Tel Aviv University, Tel Aviv 69978, Israel

${ }^{24}$ International Centre for Elementary Particle Physics and Department of Physics, University of Tokyo, Tokyo 113, and Kobe University, Kobe 657, Japan

${ }^{25}$ Institute of Physical and Environmental Sciences, Brunel University, Uxbridge, Middlesex UB8 3PH, UK

${ }^{26}$ Particle Physics Department, Weizmann Institute of Science, Rehovot 76100, Israel

${ }^{27}$ Universität Hamburg/DESY, II Institut für Experimental Physik, Notkestrasse 85, D-22607 Hamburg, Germany

${ }^{28}$ University of Victoria, Department of Physics, P O Box 3055, Victoria BC V8W 3P6, Canada

${ }^{29}$ University of British Columbia, Department of Physics, Vancouver BC V6T 1Z1, Canada

${ }^{30}$ University of Alberta, Department of Physics, Edmonton AB T6G 2J1, Canada

${ }^{31}$ Duke University, Dept of Physics, Durham, NC 27708-0305, USA

${ }^{32}$ Research Institute for Particle and Nuclear Physics, H-1525 Budapest, P O Box 49, Hungary

${ }^{33}$ Institute of Nuclear Research, H-4001 Debrecen, P O Box 51, Hungary 
${ }^{34}$ Ludwigs-Maximilians-Universität München, Sektion Physik, Am Coulombwall 1, D-85748 Garching, Germany

${ }^{a}$ and at TRIUMF, Vancouver, Canada V6T 2A3

${ }^{b}$ and Royal Society University Research Fellow

${ }^{c}$ and Institute of Nuclear Research, Debrecen, Hungary

${ }^{d}$ and Department of Experimental Physics, Lajos Kossuth University, Debrecen, Hungary

${ }^{e}$ on leave of absence from the University of Freiburg 


\section{Introduction}

Knowledge of the tau lepton properties is becoming increasingly more precise with the large data sets available. Measurements of the decay modes to a single charged particle (1-prong) and three charged particles (3-prong) have been made by numerous experiments with precision surpassing the $1 \%$ level [1]. However, only a few measurements of the $\tau^{-} \rightarrow 3 h^{-} 2 h^{+} \nu_{\tau}$ and $\tau^{-} \rightarrow 3 h^{-} 2 h^{+} \pi^{0} \nu_{\tau}$ ! branching ratios (5prong) have been made [2, 3, 4, 5]. Studies of the 5-prong decay modes are important as they are used in the determination of the mass limit on the tau neutrino (for example, see ref. [6]). Further the branching ratios of tau decays to five and six pions can be compared with the predictions of an isospin model [7], 8].

This paper presents a new measurement of these modes using the data collected between 1991 and 1995 at energies close to the $\mathrm{Z}^{0}$ resonance, corresponding to an integrated luminosity of $163 \mathrm{pb}^{-1}$, with the OPAL detector at LEP. A description of the OPAL detector can be found in ref. [9]. The performance and particle identification capabilities of the OPAL jet chamber are described in ref. [10]. The tau pair Monte Carlo samples used in this analysis were generated using the KORALZ 4.0 package [11. The dynamics of the tau decays were simulated with the TAUOLA 2.0 decay library [12]. A total of 830000 tau pair Monte Carlo events were used in this analysis. In addition, samples of $5000 \tau^{-} \rightarrow 3 h^{-} 2 h^{+} \nu_{\tau}$ and 2000 $\tau^{-} \rightarrow 3 h^{-} 2 h^{+} \pi^{0} \nu_{\tau}$ Monte Carlo events were also used. Both the $\tau^{-} \rightarrow 3 h^{-} 2 h^{+} \nu_{\tau}$ and $\tau^{-} \rightarrow 3 h^{-} 2 h^{+} \pi^{0} \nu_{\tau}$ decays were generated with a uniform phase space distribution. The $\tau^{-} \rightarrow 3 h^{-} 2 h^{+} 2 \pi^{0} \nu_{\tau}$ decay was not simulated. The Monte Carlo events were then passed through the OPAL detector simulation [13].

\section{Event selection}

The procedure used to select $\mathrm{Z}^{0} \rightarrow \tau^{+} \tau^{-}$events is similar to that described in previous OPAL publications (for example, see [14]). The $\tau^{+} \tau^{-}$events are characterized by a pair of back-to-back, narrow jets with low particle multiplicity. The two tau jets are restricted to the barrel region of the OPAL detector by requiring that the polar angle of the two jets satisfy $\overline{|\cos \theta|}<0.68$ in order to avoid regions of non-uniform calorimeter response. Background from other two fermion events is reduced by a number of requirements. Multihadronic events $\left(\mathrm{e}^{+} \mathrm{e}^{-} \rightarrow \mathrm{q} \overline{\mathrm{q}}\right)$ are significantly reduced by requiring fewer than eight tracks and ten electromagnetic clusters per event. Bhabha $\left(\mathrm{e}^{+} \mathrm{e}^{-} \rightarrow \mathrm{e}^{+} \mathrm{e}^{-}\right)$and muon pair $\left(\mathrm{e}^{+} \mathrm{e}^{-} \rightarrow \mu^{+} \mu^{-}\right)$events are removed by rejecting events where the total electromagnetic energy or the scalar sum of the track momenta are close to the centre-of-mass. Two photon $\left(\mathrm{e}^{+} \mathrm{e}^{-} \rightarrow\left(\mathrm{e}^{+} \mathrm{e}^{-}\right) \mathrm{e}^{+} \mathrm{e}^{-}\right.$or $\left.\mathrm{e}^{+} \mathrm{e}^{-} \rightarrow\left(\mathrm{e}^{+} \mathrm{e}^{-}\right) \mu^{+} \mu^{-}\right)$events are removed by rejecting events in which there is little energy in the electromagnetic calorimeter.

In the OPAL tau pair selection events are usually required to have between two and six tracks; however, events with up to eight tracks are allowed in the present selection in order to increase the efficiency for $\tau^{-} \rightarrow 3 h^{-} 2 h^{+}\left(\pi^{0}\right) \nu_{\tau}$ decays. In addition, tracks are normally required to have at least one hit in the central drift chamber at a radius of less than $75 \mathrm{~cm}$ from the beam axis. However, for this analysis this requirement is not imposed in order to avoid rejecting tracks that overlap at small radii. The efficiency for selecting tau pair events is approximately $54 \%$ from the Monte Carlo simulation, primarily due to the requirement that the tau jets are in the barrel region of the OPAL detector.

A total of $98347 \tau^{+} \tau^{-}$candidates were selected from the 1991-1995 data set. The number of $\mathrm{e}^{+} \mathrm{e}^{-} \rightarrow$

\footnotetext{
${ }^{1}$ Charge conjugation is implied throughout this paper. The symbol $h^{-}$is used to indicate either $\pi^{-}$or $\mathrm{K}^{-}$.
} 
$\mathrm{e}^{+} \mathrm{e}^{-}, \mathrm{e}^{+} \mathrm{e}^{-} \rightarrow \mu^{+} \mu^{-}$and $\mathrm{e}^{+} \mathrm{e}^{-} \rightarrow\left(\mathrm{e}^{+} \mathrm{e}^{-}\right) \mathrm{X} \quad$ (where $\mathrm{X}$ is either $\mathrm{e}^{+} \mathrm{e}^{-}$or $\mu^{+} \mu^{-}$) events in the tau pair sample is unaffected by the modifications in the standard tau pair selection and the background fraction is estimated to be $(1.24 \pm 0.09) \%$ (see ref. [14]). The $\mathrm{e}^{+} \mathrm{e}^{-} \rightarrow \mathrm{q} \overline{\mathrm{q}}$ background, however, is sensitive to the requirement on the number of tracks and was determined to be $(0.74 \pm 0.05) \%$ in the selected sample. The $q \bar{q}$ background in the tau pair sample was determined by comparing the data with the expectation of the Monte Carlo simulation [15]. The q $\bar{q}$ Monte Carlo generator is found to give more low multiplicity $q \bar{q}$ events than are observed in the data and a correction is made to the $q \bar{q}$ Monte Carlo. The total fraction of background is estimated to be $(2.0 \pm 0.1) \%$.

The $\tau^{-} \rightarrow 3 h^{-} 2 h^{+}\left(\pi^{0}\right) \nu_{\tau}$ selection begins by identifying jets with five well-measured tracks where the absolute value of the sum of the charges of the tracks must be equal to unity. Approximately $72 \%$ of the $\tau^{-} \rightarrow 3 h^{-} 2 h^{+}\left(\pi^{0}\right) \nu_{\tau}$ jets in the tau pair sample are selected with these requirements. A large fraction of this sample are $\tau^{-} \rightarrow 3 \pi^{-}\left(\geq 1 \pi^{0}\right) \nu_{\tau}$ decays where one of the photons in the final state undergoes a conversion to an $\mathrm{e}^{+} \mathrm{e}^{-}$pair. A neural network algorithm [16] found $70 \%$ of the 5 -track jets contained a photon conversion and these jets are removed from the sample. The rejection of jets with photon conversions decreases the efficiency for selecting $\tau^{-} \rightarrow 3 h^{-} 2 h^{+}\left(\pi^{0}\right) \nu_{\tau}$ jets from $72 \%$ to $64 \%$.

The sample of 5 -track jets also includes $\tau^{-} \rightarrow X^{-} \mathrm{K}_{S}^{0} \nu_{\tau}$ decays where the $\mathrm{K}_{S}^{0}$ decays to $\pi^{+} \pi^{-}$and $X^{-}$ is any number of hadrons. The sample also includes jets where a hadron has interacted in a part of the detector creating secondary particles. After the 5-track jets with photon conversions have been removed, approximately $20 \%$ of the remaining 5 -track jets are found to have a pair of oppositely charged tracks with a secondary vertex in the $r-\phi$ plane. Details of the secondary vertex algorithm can be found in [17]. The 5-track jets with an identified secondary vertex are rejected and the $\tau^{-} \rightarrow 3 h^{-} 2 h^{+}\left(\pi^{0}\right) \nu_{\tau}$ selection efficiency decreases from $64 \%$ to $62 \%$.

The residual background is reduced by requiring that each track in the jet has a momentum $(p)$ greater than $0.5 \mathrm{GeV}$ (see Fig. 1(a)) and that the invariant mass of the five tracks is less than $3 \mathrm{GeV}$ assuming that the tracks are pions (see Fig. 1(b)). The jet is rejected if the lowest momentum track has $p<2 \mathrm{GeV}$ and $\mathrm{d} E / \mathrm{d} x>9 \mathrm{keV} / \mathrm{cm}$ (see Fig. 1 (c)), where $\mathrm{d} E / \mathrm{d} x$ is the ionization energy deposited by the track in the OPAL jet chamber. The $\mathrm{d} E / \mathrm{d} x$ on a track is only considered to be reliable if there are at least 20 wires out of a possible 159 wires with good $\mathrm{d} E / \mathrm{d} x$ measurements. Background from $\mathrm{e}^{+} \mathrm{e}^{-} \rightarrow \mathrm{q} \overline{\mathrm{q}}$ events is reduced by limiting the number of clusters in the electromagnetic calorimeter to a maximum of eight clusters in the event (see Fig. 1 1 (d)). These selection criteria reduce the background to approximately $20 \%$ of the sample while only slightly decreasing the $\tau^{-} \rightarrow 3 h^{-} 2 h^{+}\left(\pi^{0}\right) \nu_{\tau}$ selection efficiency from $62 \%$ to $56 \%$.

A total of 152 five-track jets pass the selection. The sample includes a background estimated to be approximately 22 jets from other tau decays (primarily 3-prong decays) and 10 jets from q $\bar{q}$ events.

\section{Results}

The number of $\tau^{-} \rightarrow 3 h^{-} 2 h^{+} \nu_{\tau}$ and $\tau^{-} \rightarrow 3 h^{-} 2 h^{+} \pi^{0} \nu_{\tau}$ decays is determined by performing a binned likelihood fit of the $E / p$ spectrum, where $E$ is the sum of all the electromagnetic energy in the jet and $p$ is the scalar sum of the momentum of the five tracks. The shapes of the $E / p$ distributions are obtained from high statistics $\tau^{-} \rightarrow 3 h^{-} 2 h^{+} \nu_{\tau}$ and $\tau^{-} \rightarrow 3 h^{-} 2 h^{+} \pi^{0} \nu_{\tau}$ Monte Carlo samples. The $E / p$ distributions for the background are obtained from the tau and $q \bar{q}$ Monte Carlo samples. The normalization of the 


\begin{tabular}{lrr}
\hline & $\tau^{-} \rightarrow 3 h^{-} 2 h^{+} \nu_{\tau}$ & $\tau^{-} \rightarrow 3 h^{-} 2 h^{+} \pi^{0} \nu_{\tau}$ \\
\hline Events & $96.5 \pm 14.4$ & $22.6 \pm 14.8$ \\
Tau candidates & 196694 & 196694 \\
Efficiency & $0.584 \pm 0.007$ & $0.463 \pm 0.011$ \\
Non-tau background & $0.020 \pm 0.002$ & $0.020 \pm 0.002$ \\
Bias factor & $0.941 \pm 0.009$ & $0.931 \pm 0.014$ \\
\hline Branching Ratio & $(0.091 \pm 0.014 \pm 0.005) \%$ & $(0.027 \pm 0.018 \pm 0.007) \%$ \\
\hline
\end{tabular}

Table 1: The results used to calculate the branching ratios.

$\tau^{-} \rightarrow 3 h^{-} 2 h^{+} \nu_{\tau}, \tau^{-} \rightarrow 3 h^{-} 2 h^{+} \pi^{0} \nu_{\tau}$, tau background and q $\bar{q}$ background $E / p$ distributions were allowed to vary in the fit. The uncertainties in the tau and $q \bar{q}$ backgrounds (discussed below) were included into the likelihood fit. The result of the fit is shown in Fig. 3 .

The branching ratios were evaluated using

$$
B=\frac{N}{N_{\tau}(1-f)} \frac{1}{\epsilon} \frac{1}{F_{\mathrm{B}}}
$$

where $N$ is the number of signal events determined from the likelihood fit, $N_{\tau}$ is the number of tau candidates, $f$ is the non-tau background in the tau pair sample, and $\epsilon$ is the efficiency.

The tau pair selection does not select all tau decay modes with equal probability. The factor $F_{\mathrm{B}}$, obtained from Monte Carlo, corrects for the bias introduced by the tau pair selection. In general, these factors are close to unity for most tau decay channels, however, they are slightly less than unity for the $\tau^{-} \rightarrow 3 h^{-} 2 h^{+} \nu_{\tau}$ and $\tau^{-} \rightarrow 3 h^{-} 2 h^{+} \pi^{0} \nu_{\tau}$ decay channels (see Table 1) due to the requirement that the tau pair events have less than or equal to eight tracks. Minor variations in the tau pair selection criteria were found to have little impact on the value of $F_{\mathrm{B}}$.

The results of this likelihood fit are given in Table 1. The uncertainty of the background (discussed below) was included in the likelihood fit. The normalization factors of the tau and q $\bar{q}$ backgrounds obtained in the fit were $0.996 \pm 0.217$ and $1.004 \pm 0.337$, respectively. The correlation coefficient between the $\tau^{-} \rightarrow 3 h^{-} 2 h^{+} \nu_{\tau}$ and $\tau^{-} \rightarrow 3 h^{-} 2 h^{+} \pi^{0} \nu_{\tau}$ branching ratios is found to be -0.60 . The systematic errors on the branching ratios include uncertainties on the tracking, energy resolution and fit method (see Table 2).

The uncertainty in the tau and $q \bar{q}$ background includes a statistical component based on the number of data and Monte Carlo events in the background sample and a systematic component based on the modelling of the tau and q $\bar{q}$ background. The statistical uncertainty is estimated to be $20 \%$ and $30 \%$ for the tau and $q \bar{q}$ backgrounds, respectively. The systematic uncertainty is determined using a sample of events in which one of the two jets has four tracks. The composition of this sample (3-prong tau decays with a photon conversion and $\mathrm{q} \overline{\mathrm{q}}$ events) is very similar to the background found in the 5-track sample. In Fig. 2(a) the $E / p$ distribution of the 4 -track jets is plotted for events in which the other jet in the event has only one track in order to enhance the tau background. The ratio of the number of 4-track jets in the data versus Monte Carlo simulation is consistent with unity and we estimate the uncertainty on the tau background to be approximately $10 \%$ based on the statistical uncertainty of the data and Monte 
Carlo samples. In Fig. 2(b), the $E / p$ distribution is plotted when the other jet in the event has more than one track in order to enhance the $q \bar{q}$ background. Again, the ratio of the number of 4 -track jets in the data versus Monte Carlo simulation is consistent with unity and we estimate the uncertainty on the $q \bar{q}$ background to be approximately $20 \%$. The combined statistical and systematic uncertainties are found to be $22 \%$ and $36 \%$ for the tau and $\mathrm{q} \overline{\mathrm{q}}$ backgrounds, respectively.

The tracks in 5-prong tau decays are extremely collimated and the results may be sensitive to the modelling of the tracks. This was investigated by studying the distribution of the angle between each track and its nearest neighbour. The data were found to be well-modelled by the Monte Carlo simulation. This comparison was repeated with a Monte Carlo where the track parameters were smeared by $\pm 20 \%$. The branching ratios were evaluated using these samples and the change in the branching ratios was included as part of the tracking systematic error (see Table 21).

Possible differences in the modelling of the number of reconstructed tracks in the data and Monte Carlo simulation were investigated using the $\mathrm{d} E / \mathrm{d} x$ distributions of the tracks. Single tracks in the OPAL detector with momentum greater than $2 \mathrm{GeV}$ should have a maximum $\mathrm{d} E / \mathrm{d} x$ of $10 \mathrm{keV} / \mathrm{cm}$. Tracks with $\mathrm{d} E / \mathrm{d} x$ substantially above $10 \mathrm{keV} / \mathrm{cm}$ are likely due to two charged particles being reconstructed as a single track. The fraction of jets in the 5-track sample where there is at least one track with $\mathrm{d} E / \mathrm{d} x>12$ $\mathrm{keV} / \mathrm{cm}$ was found to be $0.020 \pm 0.003$ and $0.036 \pm 0.002$ in the data and Monte Carlo samples, respectively. Similar results were obtained using the 4-track sample. The difference between the data and Monte Carlo simulation was accounted for by adding a $2 \%$ uncertainty to the tracking systematic error (see Table 2).

Additional studies of the modelling of the tracks were made. For example, it is possible for $\tau^{-} \rightarrow$ $3 h^{-} 2 h^{+}\left(\pi^{0}\right) \nu_{\tau}$ jets to appear in the 4-track sample if a track failed one of the track quality requirements, such as the number of hits in the tracking chamber or the impact parameter. Branching ratios obtained using different track quality requirements were almost unchanged and therefore no additional systematic error was added.

A systematic error was added to account for the uncertainty in the modelling of the electromagnetic energy. The uncertainty in the energy scale of the electromagnetic calorimeter between the data and the Monte Carlo sample was determined to be $\pm 0.5 \%$ based on studies using tau 3-prong decays. The uncertainty in the electromagnetic energy for the 5-prong tau decays was assumed to be $\pm 1 \%$. The change in the branching ratios when the energy was scaled by $\pm 1 \%$ is included in the electromagnetic energy systematic error (see Table 2). In addition, the effect of smearing the electromagnetic energy on the branching ratios was found to be negligible.

The reliability of the likelihood fit was investigated by using the tau Monte Carlo sample to generate a many sets of 'data' and subsequently fitting it with the distributions obtained from the high statistic signal Monte Carlo samples. The systematic error quoted on the fit is the result of changing the upper range of the fit (nominally $E / p=1.2$ ) between $E / p=0.9$ and 1.4 .

The $\tau^{-} \rightarrow 3 h^{-} 2 h^{+} \nu_{\tau}$ and $\tau^{-} \rightarrow 3 h^{-} 2 h^{+} \pi^{0} \nu_{\tau}$ branching ratios were determined assuming that the $\tau^{-} \rightarrow 3 h^{-} 2 h^{+} 2 \pi^{0} \nu_{\tau}$ branching ratio was negligible. The CLEO Collaboration obtained a limit on the $\tau^{-} \rightarrow 3 h^{-} 2 h^{+} 2 \pi^{0} \nu_{\tau}$ decay of $0.011 \%$ [3], suggesting that this decay mode could contribute up to $10 \%$ of the inclusive 5-prong branching ratio (based on the CLEO branching ratios). The systematic error due 


\begin{tabular}{lrr}
\hline & $\tau^{-} \rightarrow 3 h^{-} 2 h^{+} \nu_{\tau}(\%)$ & $\tau^{-} \rightarrow 3 h^{-} 2 h^{+} \pi^{0} \nu_{\tau}(\%)$ \\
\hline Tracking & 0.004 & 0.006 \\
Electromagnetic energy & 0.002 & 0.001 \\
Fit & 0.000 & 0.003 \\
$\tau^{-} \rightarrow 3 h^{-} 2 h^{+} 2 \pi^{0} \nu_{\tau}$ & 0.000 & 0.003 \\
\hline Total & 0.005 & 0.007 \\
\hline
\end{tabular}

Table 2: The systematic uncertainties of the $\tau^{-} \rightarrow 3 h^{-} 2 h^{+} \nu_{\tau}$ and $\tau^{-} \rightarrow 3 h^{-} 2 h^{+} \pi^{0} \nu_{\tau}$ branching ratios.

to this assumption is given in Table 2 and was determined by assuming that the efficiency for selecting $\tau^{-} \rightarrow 3 h^{-} 2 h^{+} 2 \pi^{0} \nu_{\tau}$ decays is approximately 0.3 and that one third of the $\tau^{-} \rightarrow 3 h^{-} 2 h^{+} \pi^{0} \nu_{\tau}$ candidates are $\tau^{-} \rightarrow 3 h^{-} 2 h^{+} 2 \pi^{0} \nu_{\tau}$ decays.

The inclusive 5-prong branching, $\tau^{-} \rightarrow 3 h^{-} 2 h^{+}\left(\geq 0 \pi^{0}\right) \nu_{\tau}$, was determined by applying the same selection procedure used in the exclusive measurement. The inclusive branching ratio was determined using

$$
B=\frac{N-N_{\mathrm{q} \overline{\mathrm{q}}}-N_{\tau}^{b k g d}}{N_{\tau}(1-f) \epsilon F_{\mathrm{B}}}
$$

where $N$ is the number of 5 -track jets in the sample (149.0), $N_{\mathrm{q} \overline{\mathrm{q}}}$ is the number of background jets from $\mathrm{q} \overline{\mathrm{q}}$ events $(8.7), N_{\tau}^{b k g d}$ is the number of background jets from tau decays $(21.3), \epsilon$ is the efficiency for selecting the signal jets $(0.556)$, and $F_{\mathrm{B}}$ is the bias factor $(0.94 \pm 0.01)$. The branching ratio is found to be $(0.119 \pm 0.013 \pm 0.008) \%$ where the first error is statistical and the second is systematic. The systematic errors are similar to those discussed above. The error on the efficiency includes a statistical component (0.006), a component (0.020) associated with the uncertainty in the ratio between the $\tau^{-} \rightarrow 3 h^{-} 2 h^{+} \nu_{\tau}$ and $\tau^{-} \rightarrow 3 h^{-} 2 h^{+} \pi^{0} \nu_{\tau}$ branching ratios quoted here and a component (0.030) for the possible contribution from the $\tau^{-} \rightarrow 3 h^{-} 2 h^{+} 2 \pi^{0} \nu_{\tau}$ decay (discussed below).

The inclusive branching ratio was calculated assuming that the efficiency for selecting $\tau^{-} \rightarrow 3 h^{-} 2 h^{+} 2 \pi^{0} \nu_{\tau}$ decays is the same as the efficiency quoted above. However, the efficiency for selecting $\tau^{-} \rightarrow 3 h^{-} 2 h^{+} \nu_{\tau}$ and $\tau^{-} \rightarrow 3 h^{-} 2 h^{+} \pi^{0} \nu_{\tau}$ decays was found to 0.58 and 0.46 , respectively, so this assumption may not be valid. The difference in the $\tau^{-} \rightarrow 3 h^{-} 2 h^{+} \nu_{\tau}$ and $\tau^{-} \rightarrow 3 h^{-} 2 h^{+} \pi^{0} \nu_{\tau}$ efficiencies was found to be due to the requirement that there be five tracks in each candidate jet. The $\tau^{-} \rightarrow 3 h^{-} 2 h^{+} \pi^{0} \nu_{\tau}$ decays contain a $\pi^{0}$ that can produce additional tracks by a Dalitz decay or photon conversion. The distribution of the number of electromagnetic clusters was found to have the same shape for the $\tau^{-} \rightarrow 3 h^{-} 2 h^{+} \nu_{\tau}$ and $\tau^{-} \rightarrow 3 h^{-} 2 h^{+} \pi^{0} \nu_{\tau}$ so the cut on this quantity is not a concern; this is not unexpected as tau decays are highly collimated and a coarse clustering algorithm is used. The systematic error on our efficiency $(0.030)$ was obtained by assuming that $10 \%$ of the inclusive branching ratio is due to $\tau^{-} \rightarrow 3 h^{-} 2 h^{+} 2 \pi^{0} \nu_{\tau}$ decays (based on the CLEO branching ratios) and that the efficiency for selecting these decays is approximately 0.30 . 


\section{Summary}

The branching ratios of the decay of the $\tau$ lepton to five charged particles are found to be

$$
\begin{gathered}
B\left(\tau^{-} \rightarrow 3 h^{-} 2 h^{+} \nu_{\tau}\right)=(0.091 \pm 0.014 \pm 0.005) \% \\
B\left(\tau^{-} \rightarrow 3 h^{-} 2 h^{+} \pi^{0} \nu_{\tau}\right)=(0.027 \pm 0.018 \pm 0.007) \%
\end{gathered}
$$

where the first error is statistical and the second systematic. These results are in good agreement with previous measurements [2, 3, 4, 5]. These results when combined with other measurements of tau decays to five pions are found to be consistent with the prediction of an isospin model [0, 8].

\section{Acknowledgements:}

We particularly wish to thank the SL Division for the efficient operation of the LEP accelerator at all energies and for their continuing close cooperation with our experimental group. We thank our colleagues from CEA, DAPNIA/SPP, CE-Saclay for their efforts over the years on the time-of-flight and trigger systems which we continue to use. In addition to the support staff at our own institutions we are pleased to acknowledge the

Department of Energy, USA,

National Science Foundation, USA,

Particle Physics and Astronomy Research Council, UK,

Natural Sciences and Engineering Research Council, Canada,

Israel Science Foundation, administered by the Israel Academy of Science and Humanities,

Minerva Gesellschaft,

Benoziyo Center for High Energy Physics,

Japanese Ministry of Education, Science and Culture (the Monbusho) and a grant under the Monbusho International Science Research Program,

German Israeli Bi-national Science Foundation (GIF),

Bundesministerium für Bildung, Wissenschaft, Forschung und Technologie, Germany,

National Research Council of Canada,

Research Corporation, USA,

Hungarian Foundation for Scientific Research, OTKA T-016660, T023793 and OTKA F-023259. 


\section{References}

[1] R. M. Barnett et al., Phys. Rev. D54 (1996) 1 and 1997 off-year partial update for the 1998 edition available on the PDG WWW pages (URL: http://pdg.lbl.gov/).

[2] ALEPH Collaboration, D. Buskulic et al., Z. Phys. C70 (1996) 579.

[3] CLEO Collaboration, G. Dibaut, Phys. Rev. Lett. 73 (1994) 934.

[4] ARGUS Collaboration, H. Albrecht et al., Phys. Lett. B202 (1988) 149.

[5] HRS Collaboration, B.G. Bylsma et al., Phys. Rev. D35 (1987) 2269.

[6] OPAL Collaboration, K. Ackerstaff et al., CERN-EP/98-055, 15th April 1998.

[7] R.J. Sobie, Z. Phys. C69 (1995) 99.

[8] A. Rougé, Z. Phys. C70 (1996) 65.

[9] OPAL Collaboration, K. Ahmet et al., Nucl. Instr. and Meth. A305 (1991) 275;

P.P. Allport et al., Nucl. Instr. and Meth. A324 (1993) 34;

P.P. Allport et al., Nucl. Instr. and Meth. A346 (1994) 476.

[10] M. Hauschild et al., Nucl. Instr. and Meth. A314 (1992) 74;

O. Biebel et al., Nucl. Instr. and Meth. A323 (1992) 169;

M. Hauschild, Nucl. Instr. and Meth. A379 (1996) 436.

[11] S. Jadach, B. F. L. Ward and Z. Was, Comp. Phys. Comm. 79 (1994) 503.

[12] R. Decker, S. Jadach, J. H. Kühn and Z. Was, Comp. Phys. Comm. 76 (1993) 361.

[13] J. Allison et al., Nucl. Instr. and Meth. A317 (1992) 47.

[14] OPAL Collaboration, G. Alexander et al., Phys. Lett. 369 (1996) 163.

[15] T. Sjöstrand, Comp. Phys. Comm. 82 (1994) 74.

[16] OPAL Collaboration, G. Alexander et al., Z. Phys. C70 (1996) 357.

[17] OPAL Collaboration, R. Akers et al., Phys. Lett. 339 (1994) 278. 

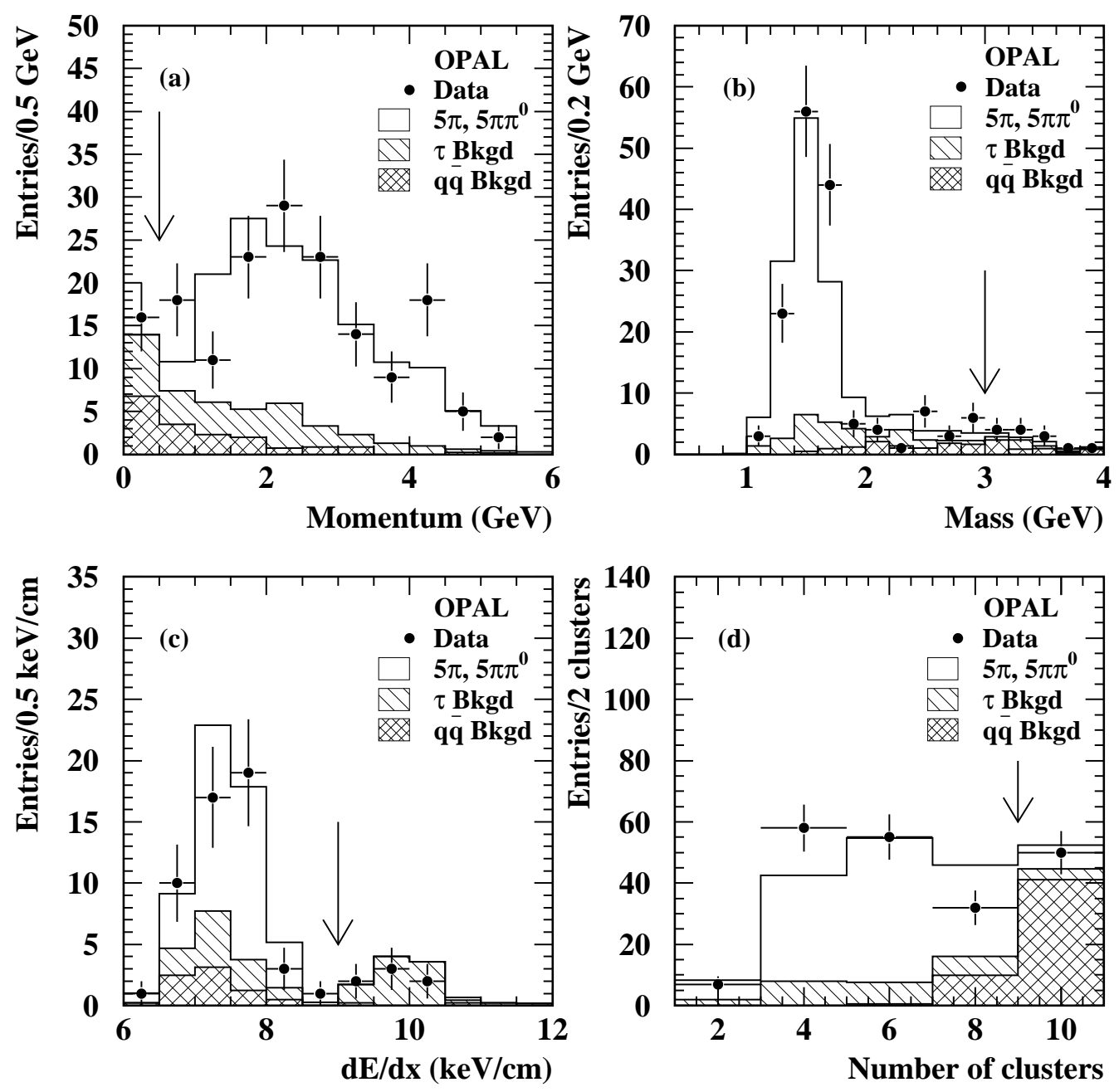

Figure 1: (a) the momentum of the lowest momentum track in the jet, (b) the mass of the five tracks, (c) the energy loss $(\mathrm{d} E / \mathrm{d} x)$ in the central drift chamber for tracks with $p<2 \mathrm{GeV}$ and (d) the number of clusters in the event. The figures are for jets selected as $\tau^{-} \rightarrow 3 h^{-} 2 h^{+}\left(\pi^{0}\right) \nu_{\tau}$ candidates. All $\tau^{-} \rightarrow 3 h^{-} 2 h^{+}\left(\pi^{0}\right) \nu_{\tau}$ selection criteria are applied and the arrows indicate the cut locations. In all plots the world average branching ratios [1] are used except for the $\tau^{-} \rightarrow 3 h^{-} 2 h^{+} \nu_{\tau}$ and $\tau^{-} \rightarrow 3 h^{-} 2 h^{+} \pi^{0} \nu_{\tau}$ decay modes where the branching ratios obtained in this work are used. 

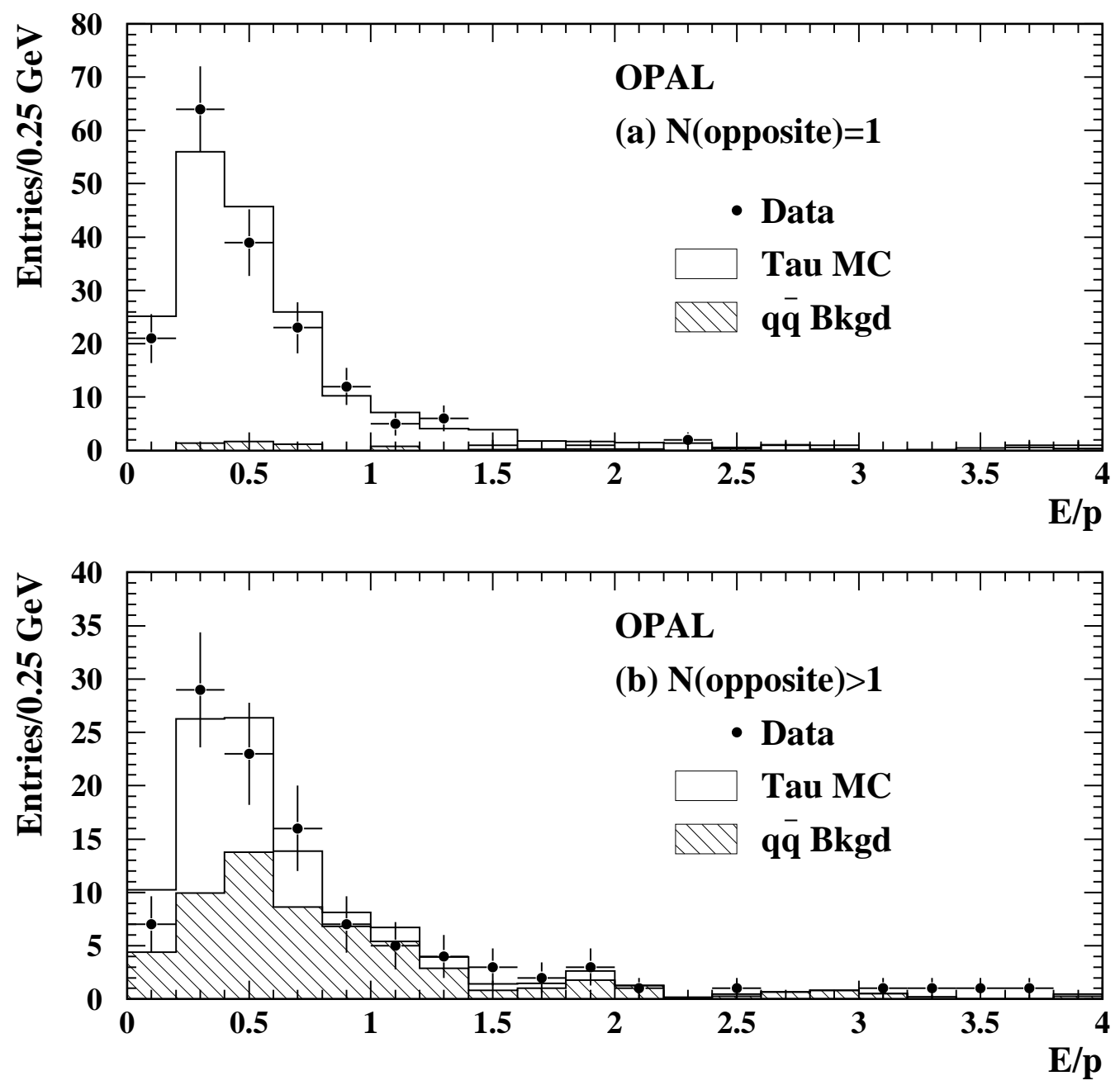

Figure 2: The $E / p$ spectrum for jets with four tracks is shown. In plot (a) there is a jet on the opposite side with only one track. In plot (b) there is a jet on the opposite side with more than one track. 


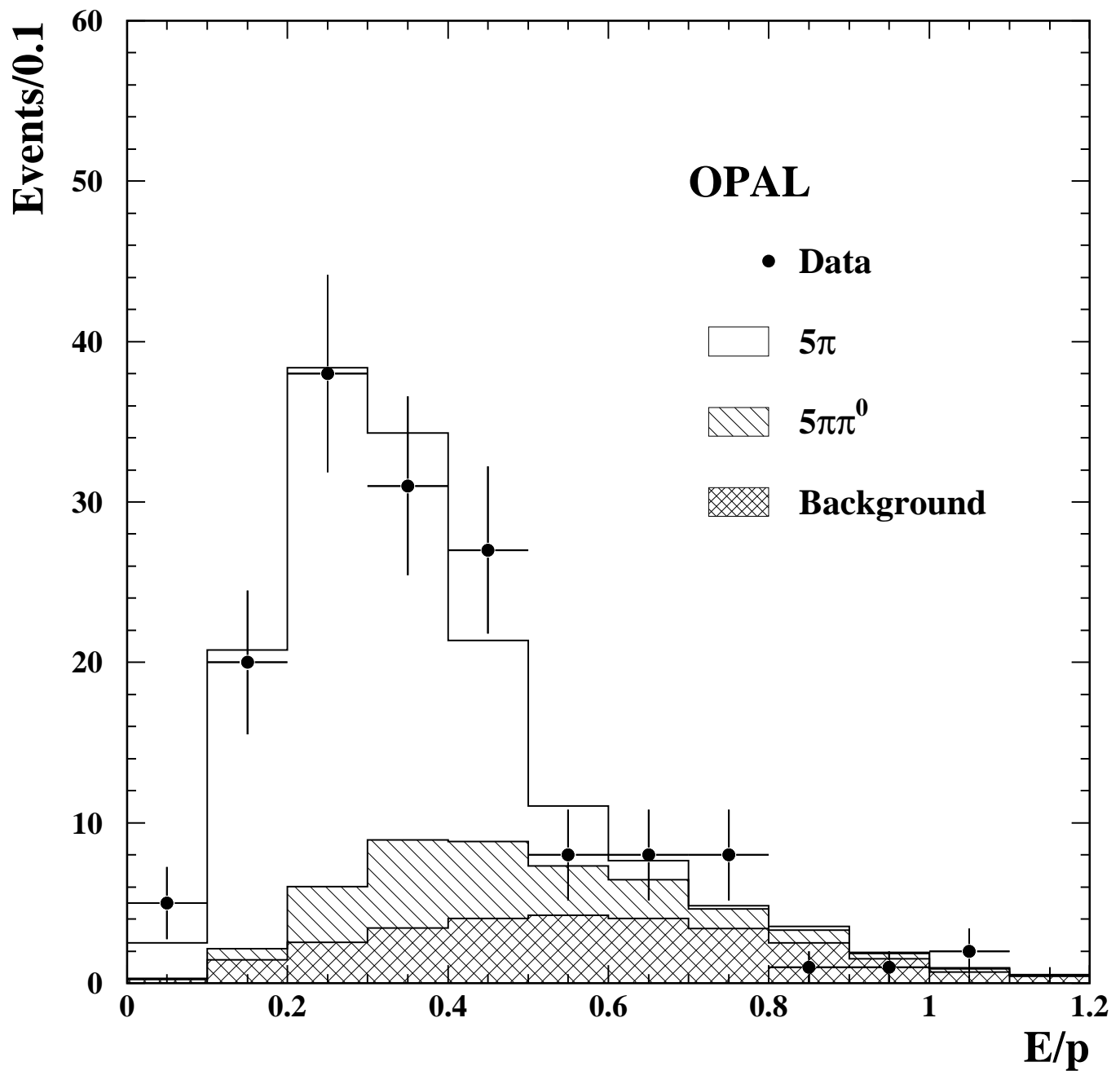

Figure 3: The $E / p$ spectrum for $\tau^{-} \rightarrow 3 h^{-} 2 h^{+}\left(\pi^{0}\right) \nu_{\tau}$ jets is shown. The histograms are the result of the fit to the data. 